\title{
REPLAY: It's March 11th. Let's try to fight Covid differently. How would you do it?
}

\author{
By: Shrey Jain (D)
}

Suppose you have the ability to go back to March 11 th of 2020 - the date the WHO officially declared COVID-19 a pandemic ${ }^{1}$.

What could have been done differently with respect to digital health product development?

Were domain experts utilized to the best of their abilities? What can we learn from COVID-19 for future public health crises planning?

Digital health data is now, more than ever, deeply influencing our lives. Having robust digital health tools to support public health surveillance is no longer a problem for technocrats.

It's everyone's business now.

Through the COVID-19 pandemic, I had the opportunity to lead a digital health startup, Flatten.ca, that focused on collecting symptom data from people in Canada and in Somalia ${ }^{2}$. This experience led to collaborations with big-tech companies, researchers, government officials, startups, and investors.

Reflecting back on my experiences, I see many flaws in the process by which the North-American community decided to develop and deploy digital health tools. Simply put, a lack of speed and strategically allocated domain expertise hindered our success. Why did Canada take 5 months to launch a contact tracing app and Singapore only 10 days?

This perspective piece aims at answering the questions highlighted above and analyzing the North-American response to build digital health tools as the COVID-19 pandemic rages on. Whether you're an investor, academic, student, founder, or otherwise involved in the digital health industry, and you're thinking about how to effectively support product development for future public health crises, this can help better inform where your efforts are best spent.

\section{WHAT IS DIGITAL HEALTH?}

Digital health is technology that is made available for society to enhance the efficiency of healthcare delivery and make medicine more personalized and precise ${ }^{3}$. COVID-19 wreaked havoc on healthcare systems, drastically affecting efficiency and thus required support from data gathered through digital health tools to effectively optimize healthcare strategies.

The onset of the COVID-19 pandemic created a call to action for startups, academics, big tech, and most importantly government bodies to each find their own ways to collect data in order to better inform rapid response efforts.

The focus for this perspective piece is on disease surveillance digital health tools: contact tracing, syndromic surveillance (symptom tracking), quarantine compliance monitoring, and flow modelling (movement tracking $)^{3}$.

\section{STAKEHOLDER ANALYSIS}

\section{Research/Academic Community:}

Academics and academic institutions immediately responded to the call to action around COVID-19.

Public health response for Ebola demonstrated that contact tracing served as an effective strategy to mitigate the spread of the virus ${ }^{4}$. There was a need to deploy a contact tracing platform in Canada as soon as possible.

Academics, not traditionally involved in leading the development and deployment of scalable products, acquired funding and human capital to build contact tracing platforms. Here in Canada, many researchers from the University of Toronto and Mila started creating contract tracing platforms using machine learning ${ }^{5,6}$. Other organizations across the country also invested in the development of contact tracing platforms ${ }^{7,8}$. 
The benefit of the academic institutions building digital health products is unclear, as they did not see widespread adoption. The success of a digital health tool was dependent not only on their ethical or legal compliance, but also support from government, and mainstream media. In the end, the Canadian government decided to work with big-tech, Google, Apple, and Shopify, for its federal contact tracing platform.

Despite academics being unable to succeed in product development and deployment, many were involved in advising technical teams of successful digital health tools in Canada. Additionally, there has been an unprecedented number of research publications on the virus and associated response efforts, and publications venues even went as far as creating new submission and acceptance programs to speed up research ${ }^{9}$.

We have seen the interconnectedness between the virus and its impact on research not only in science, but also in economics, culture, psychology, and policy. Tan Eng Chye, the current President for the National University of Singapore recognizes the value that cross-disciplinary research has led to in Singapore, to "overhaul the academic system to promote collaborations"10.

The value of this cross-disciplinary research also remains unclear. Although interested in supporting the COVID-19 pandemic, was there an additional motive for academics to simply push out COVID-19 papers to acquire grant funding to support their other work? What level of quality is the science that was produced and how much of it was useless noise?

\section{Research Community Takeaways:}

- Avoid building products without strong government support and clear growth strategies (ideally experience scaling products). I think it is important for academics and/or researchers to innovate, but when we analyze the success of the products that were built through the pandemic, widespread adoption of these products was sparse. Consider where domain expertise is best used for short-term impact.

- Cross-disciplinary efforts can be tremendously valuable for a research lab and for informing public policy.

- Supporting and advising startups/government bodies/student projects is a great way to add value. If you are in the Toronto community, connecting with Creative Destructive Labs, Next, and University Incubator programs are great places to connect with. Similar startup ecosystems are growing in every city, for example Founder Fuel in Montreal, and Velocity in Waterloo.

\section{Investors:}

Rightfully so, billions of dollars were poured into grants and funding to organizations to support developing digital health products. Jack Dorsey announced early on in the pandemic that he would be allocating $28 \%$ of his net worth to COVID-19 response efforts ${ }^{11}$.

Funding was being distributed around so fast causing rapid diligence reviews. This resulted in a tremendous misallocation of money, and a large percentage of COVID-19 projects failing to achieve their stated goals. How can we improve for next time? Now that we understand the relevance of domain experts in the broader COVID-19 battle, we can accurately distribute funds based on previous success. For example, funding academic digital health products has significantly more risk towards big-tech execution of digital health tools (as we saw through COVID-19).

There are two types of investors through the pandemic: - Government bodies/Grant Funding: organizations looking to support small business/other companies to innovate/support COVID-19 responses.

- VC/Angels: individuals/organizations looking to generate outsized returns through funding startups.

In April of 2020, the Government of Canada invested $\$ 54.2$ million into COVID-19 projects $^{12}$. Most of the government funding was allocated towards research. The impact that this funding has had towards mitigating the spread of COVID-19 in Canada is not measurable at this time.

From the Angel/VC perspective, the Creative Destructive Labs created the Recovery program early on focused towards COVID-19 early stage ventures ${ }^{13}$. NGen also supported early stage companies by allocating $\$ 50$ million to support startups working on COVID-19 related projects ${ }^{14}$.

It is still too early to understand the return on investment into these startups working on COVID-19 specific projects (ie. contact tracing / symptom monitoring). However, investment into telemedicine and "long-term" (beyond COVID-19) digital health products is very promising and is likely to generate outsized returns long-term ${ }^{15}$.

Keith Rabois has noted on TWiStartups some key notes about virtual communication as a VC/Angel: individuals get easily distracted on Zoom calls (not $100 \%$ locked in); delays in communication "hand-offs"; hard to connect with individuals you don't already know. Founders are going to have to adapt to give their pitches and connect with VC/Angels in new innovative ways to acquire funding ${ }^{16}$. 
Government Investment Takeaways:

- Founder/organization characterization is a critical component to evaluating success of the project.

- Consider asking the following questions: How well does this investment fit into the broader thesis of this academic organization?; How can we ensure that this investment aligns closely with our efforts?; Do we have individuals from our sub-team to work with these teams consistently to ensure alignment with government run strategies? Does this lab/ organization have the expertise to legitimately contribute to this problem?

VC/Angel Investment Takeaways:

- The job of a VC/Angel is to make calculated bets. COVID-19 led to rapid virtual investments. Risk is heightened but outsized returns into companies adapting to behavior change can be quite high (ie. Zoom, telehealth).

- Be clear about your objective as an investor: shortterm impact vs outsized returns. Investing in a company that returns $1 \mathrm{x}$ but helps public health tremendously does not hurt your portfolio, it in fact benefits your public persona, so why not invest in crisis response teams?

\section{Founders/Startups:}

Through the COVID-19 pandemic, startups either suffered or grew tremendously. We saw many pivot/ lean into digital health and support data collection and analysis for the COVID-19 pandemic in a variety of domains.

Recently, Darwin Al, a company centered around automated vehicles has turned their focus towards COVID-19 machine learning classification models, enabling them to do their seed round at $\$ 8$ million valuation and anticipates doing their series $A$ in Q4 of $2021^{17}$. Is this a sign of success or following hype? This company has demonstrated successful financial gains, but it is still unclear the value added/impact to supporting public health response.

COVID-19 has fundamentally impacted human behavior globally. This change means rapid innovation and rapid growth across industries, of which McKinsey notes of utmost importance in "Why Innovation is critical in times of crises"18.

\section{Key Takeaways:}

- Lean into behavior changes early.

- Support government bodies if you have the runway to do so long-term. Crisis response always lasts longer than you anticipate (the COVID-19 pandemic would be resolved far sooner than reality has provided).

\section{Governments:}

Unfortunately, the government is not able to move at the same pace as other stakeholders. In Canada, it took nearly 5 months for a product (contact tracing platform) to be deployed. This also, unfortunately, significantly impacted user engagement with the product. Early on in the pandemic, there was fear from citizens, a want to "get answers" from the government, and like me, I believe many Canadians were glued to blogs/news/ media channels trying to learn more.

What does an ideal government product roll out look like? Trace Together in Singapore had nearly $80 \%$ of the population adopt the platform through the pandemic. They launched the tool within 10 days of the pandemic being declared ${ }^{19}$. This has proven to be effective by reducing the time it takes to identify someone as being "in close contact" with a COVID-19 positive case from four days down to two ${ }^{20}$. TraceTogether launched very early. They had problems with the software, but iterated continuously and acquired strong user adoption early on. It is likely Canada spent significant time through privacy review which hindered adoption long-term and ultimately utility of the tool. What is of more value: potential privacy threats or gains from contact tracing platforms?

During the pandemic, people started to get "COVID-19 fatigue". This impacted user engagement tremendously ${ }^{21}$. Digital health tools, like contact tracing, are only effective based on the population size that uses them. In Canada, this has not been a successful launch.

It would have been optimal for big-tech companies with experience in healthcare to build and deploy digital health tools rapidly, with strong algorithmic auditing boards and have government bodies focus on strategic investment, communication with the population, and supporting healthcare systems more effectively.

\section{Big-Tech/Large Corporations:}

Large corporations and big-tech (ie. Google, Apple, Amazon, etc) have tremendous power to support public health response with respect to digital health tool development and deployment.

I believe that these organizations have the capability to support more than they did early on in the pandemic to engage a wider user base. Big-tech played a critical role in COVID-19 response, and could potentially do even more with government support. Here are some of the "good" things they contributed towards:

- In North America, the most widely adopted tools were ones that were developed by big-tech ${ }^{22,23}$. This included the collaboration between Shopify/ Google/Apple for the COVID-Alert app in Canada ${ }^{24}$. 


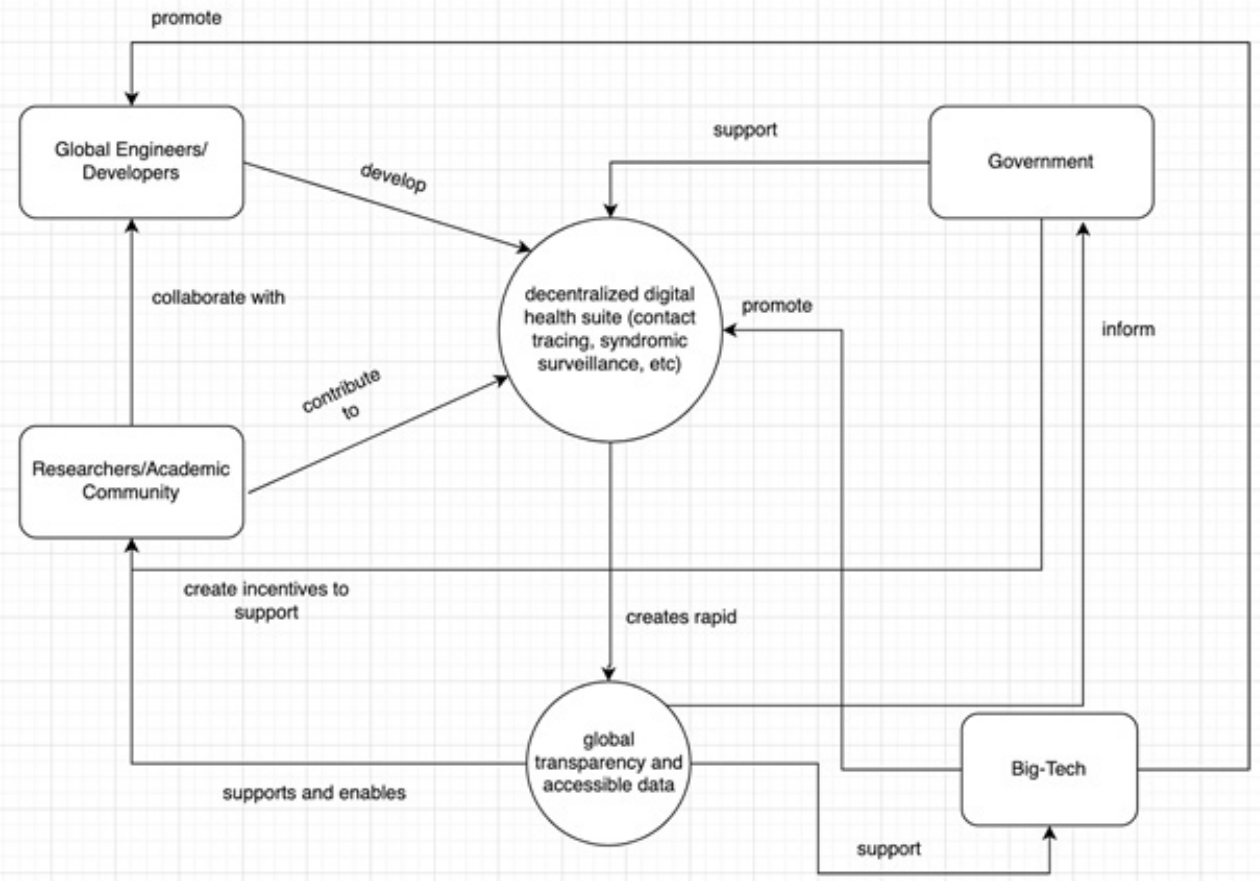

Figure 1. A proposed method for decentralized development of digital health products for COVID-19 crises response.

- Big-tech supported outside organizations to develop and innovate products for other stakeholders ${ }^{25,26}$.

Does the stigma of data privacy limit big-tech to invest heavily in COVID-19 response? Enabling big-tech organizations through government support would have been tremendously more effective to develop and deploy digital health tools faster. This in turn could also enable big-tech to build software services and optimization strategies for more effective vaccine distribution chains and enable government bodies to continue to work on policy and messaging to the broader community.

A potential alternative to existing solutions could be the development of a decentralized contact tracing platform/digital health suite of products (inclusive of syndromic surveillance, contact tracing, etc).

The lack of trust in government and large corporations with respect to privacy created tremendous friction for widespread adoption. Decentralized systems, see Figure 1, can help mitigate the fear of large corporations managing data. We are seeing cryptocurrency growing in popularity due to its benefits with respect to privacy, and for future crises, maybe this is the best way to gain the public's trust and rapid adoption.

\section{Summary:}

Without a reflection on our efforts through COVID-19, how can we ever expect to improve response efforts for future crises. The COVID-19 pandemic has tested people, across all domains and industries, of which mistakes were bound to be made. It is our responsibility to learn, iterate, and change for the future.

\section{Future Questions:}

- How can we improve cross-disciplinary communication to develop these tools to avoid mass reproduction and waste of time?

- Is there a utilitarian way to analyze this effort? le. If you are an NBA coach, you put the players in the roles they play best, how can we do the same for crisis response with domain experts? Do we even need to?

- How should biotech companies be involved in digital health tool surveillance?

- When rolling out digital health products, should we also consider long-term crisis planning efforts (ie. vaccine distribution chains)?

- What do the lessons from COVID-19 teach us about our potential collaborative engagement to fight the climate crises?

Shrey Jain is a student in the Department of Engineering Science at the University of Toronto, a member of the Vector Institute of Technology (Health Machine Learning Research Group), and the President of Flatten. Please address correspondence to: shreyd. jain@mail.utoronto.ca

\section{ACKNOWLEDGEMENTS}

Thank you to Martin Staadecker, Arthur Allshire, Ben 
Agro, Amanda Caromicoli, Nick Frosst, and Taylor Killian for reviewing and reading through this piece.

\section{COMPETING INTERESTS}

No competing interests declared.

Received: January 22, 2021

Accepted: February 22, 2021

Published online: March 22, 2021

\section{REFERENCES}

1. WHO Director-General's opening remarks at the media briefing on COVID-19 - 11 March 2020. https://www.who.int/directorgeneral/speeches/detail/who-director-general-s-opening-remarksat-the-media-briefing-on-covid-19---11-march-2020.

2. Home. https://www.flatten.ca/.

3. Gasser, U., lenca, M., Scheibner, J., Sleigh, J. \& Vayena, E. Digital tools against COVID-19: taxonomy, ethical challenges, and navigation aid. Lancet Digit Health 2, e425-e434 (2020).

4. Saurabh, S. \& Prateek, S. Role of contact tracing in containing the 2014 Ebola outbreak: a review. Afr. Health Sci. 17, 225-236 (2017).

5. Martineau, V. Yoshua Bengio and his collaborators unveil the name of their COVID-19 tracing application and launch a White Paper. https://mila.quebec/en/yoshua-bengio-and-his-collaboratorsunveil-the-name-of-their-covid-19-tracing-application-and-launcha-white-paper/ (2020).

6. $U$ of $T$ researcher aims to improve accuracy of COVID-19 contact tracing with MyTrace app. https://www.utoronto.ca/news/ u-t-researcher-aims-improve-accuracy-covid-19-contact-tracingmytrace-app.

7. Ray.health. http://ray.health/.

8. ABTraceTogether. https://www.alberta.ca/ab-trace-together. aspx.

9. COVID-19 research update: How many pandemic papers have been published? https://www.natureindex.com/news-blog/howcoronavirus-is-changing-research-practices-and-publishing.

10. COVID-19 starts push for more interdisciplinary research.https://www.universityworldnews.com/post. php?story=20200724125405567.

11. Statt, N. Jack Dorsey to donate $\$ 1$ billion to fund COVID-19 relief and other charities. https://www.theverge.com/2020/4/7/21212766/ jack-dorsey-coronavirus-covid-19-donate-relief-fund-square-twitter 12. Canadian Institutes of Health Research. Government of Canada funds 49 additional COVID-19 research projects - Details of the funded projects. Government of Canada https://www.canada. ca/en/institutes-health-research/news/2020/03/government-ofcanada-funds-49-additional-covid-19-research-projects-details-ofthe-funded-projects.html (2020).

13. Recovery - creative destruction lab. https://www. creativedestructionlab.com/program/recovery/.

14. NGen. NGen. https://www.ngen.ca/covidprojectguide.

15. American Medical Association. Telehealth's post-pandemic future: Where do we go from here? https://www.ama-assn.org/ practice-management/digital/telehealth-s-post-pandemic-futurewhere-do-we-go-here.
16. This week in startups. https://thisweekinstartups.com/ (2010). 17. Simpson, M. Today in funding: RockMass technologies, DarwinAl. https://betakit.com/today-in-funding-rockmasstechnologies-darwinai/ (2020).

18. Am, J. B., Furstenthal, L., Jorge, F. \& Roth, E. Innovation in a crisis: Why it is more critical than ever. https://www.mckinsey.com/ business-functions/strategy-and-corporate-finance/our-insights/ innovation-in-a-crisis-why-it-is-more-critical-than-ever (2020).

19. Singapore government launches new app for contact tracing to combat spread of COVID-19. https://www.mobihealthnews. com/news/asia-pacific/singapore-government-launches-new-appcontact-tracing-combat-spread-covid-19 (2020).

20. Wikipedia contributors. TraceTogether. Wikipedia, The Free Encyclopedia https://en.wikipedia.org/w/index. php?title=TraceTogether\&oldid=999988659 (2021).

21. Kirkey, S. COVID-19 quarantine fatigue: The arrival of summer will test our resolve to social distance. https://www.saltwire. com/lifestyles/health/covid-19-quarantine-fatigue-the-arrival-ofsummer-will-test-our-resolve-to-social-distance-453174/.

22. Pichai, S. Coronavirus: How we're helping. Google https:// blog.google/inside-google/company-announcements/coronaviruscovid19-response/ (2020).

23. Apple expands partnership with (RED) to combat HIVIAIDS and COVID-19. Apple https://www.apple.com/ca/newsroom/2020/12/ apple-expands-partnership-with-red-to-combat-hiv-aids-andcovid-19/ (2020).

24. Ontario Newsroom. https://news.ontario.ca/en/release/57862/ covid-alert-available-for-download-beginning-today.

25. Home. https://www.gov.sg/.

26. Pichai, S. COVID-19: $\$ 800+$ million to support small businesses and crisis response. Google https://blog.google/inside-google/ company-announcements/commitment-support-small-businessesand-crisis-response-covid-19/ (2020). 